\title{
Effect of Hamstring Stretching on Pelvic Angle and Biofeedback Pressure during Passive Straight Leg Raising
}

\author{
Do-young Jung, PT, Ph.D
}

Department of Physical Therapy, College of Tourism \& Health, Kinesiopathologic Science Institute, Joongbu University, Geumsan, South Korea

Background In clinical settings, a pressure biofeedback unit (PBU) is sometimes used to detect pelvic movement and retrain movement patterns during lower limb movement. Hamstring length influences changes in pelvic movement during passive straight leg raising (PSLR).

Purpose To determine the relationship between pelvic motion and biofeedback pressure measured by a PBU during PSLR, and to identify changes in biofeedback pressure after hamstring stretching.

Study design Cross-sectional study

Methods Twenty participants with short hamstrings were recruited for this study. A three-dimensional motion analysis system and PBU were used to measure pelvic angle and biofeedback pressure during PSLR, respectively. The pelvis angle and biofeedback pressure were measured before and after hamstring stretching, increasing PSLR in 10-degree increments.

Results There was a significant relationship between pelvic motion and biofeedback pressure (Pearson's $r=0.76, p<0.05$ ). Linear regression equation using lumbopevic angle as an independent factor was as follows: Pressure of $\mathrm{PBU}=45.05+(3.35 \times$ angle of lumbopelvic motion $)\left(\mathrm{R}^{2}=0.57\right.$, $p<0.05)$. Biofeedback pressure decreased significantly between PSLR angles of $10-60^{\circ}$ after hamstring stretching $(p<0.05)$. The pelvic angle decreased significantly at PSLR angles of $50^{\circ}$ and $60^{\circ}$ after hamstring stretching $(p<0.05)$.

Conclusions Biofeedback pressure measured with a PBU can be used to assess pelvic motion during muscle length tests following hamstring stretching.

Key words Biofeedback pressure; Pelvic motion; Straight leg raise.

\author{
J Musculoskelet \\ Sci Technol \\ 2021; 5(2): 47-53 \\ Published Online \\ Dec 31, 2021 \\ pISSN 2635-8573 \\ eISSN 2635-8581 \\ Article History \\ Received 30 Aug 2021 \\ Revised 12 Sep 2021 \\ (1st) \\ Revised 15 Sep 2021 \\ (2nd) \\ Accepted 17 Sep 2021

\section{CONTACT} \\ ptsports@joongbu.ac.kr \\ Do-young Jung, \\ Department of Physical \\ Therapy, College of \\ Tourism \& Health, \\ Kinesiopathologic \\ Science Institute, \\ Joongbu University, \\ Geumsan, South Korea \\ This is an Open-Access article \\ distributed under the terms of \\ the Creative Commons Att- \\ ribution Non-Commercial Li- \\ cense (http:/creativecommons. \\ cense (hittp./creativecommons. \\ org/icenses/by-nc/4.0) whic \\ pernits unrestricted non-co- \\ mercial use, distribution, \\ and reproduction in any me- \\ dium, provided the origina \\ work is properly cited.
}

\section{INTRODUCTION}

During hip flexion, it is important to distinguish femoral motion from pelvic motion. ${ }^{1-2}$ Because hip joint and pelvis move sequentially during hip flexion, clinicians commonly attempt to detect the onset of pelvic rotation when measuring the range of hip flexion, or to limit pelvic movement when measuring hamstring muscle length. ${ }^{1,3}$ However, previous studies have demonstrated concurrent movement of the femur and pelvis, posterior tilting of the pelvis, and flattening of the lumbar spine during hip flexion; collec- tively, these movements are termed pelvifemoral rhythm. ${ }^{4-10}$ The contribution of pelvic rotation to hip flexion onsets at a very early point and then persists throughout the range of hip flexion. ${ }^{4-10}$ Thus, clinicians must consider simultaneous pelvifemoral rhythm when measuring the range of hip flexion or hamstring muscle length.

In previous studies, pelvic motion accompanying hip flexion was analyzed using various tools, including tape lines ${ }^{4-6}$ or tape markers, ${ }^{9}$ spherical reflective markers, ${ }^{7,8,10}$ and magnetic sensors. ${ }^{11}$ However, these tools require time and effort for installation and analysis of the acquired data, 
and are not easy to apply in clinical settings. Although observation and palpation of the pelvis have been commonly used to measure the range of hip flexion or hamstring muscle length, its sensitivity is insufficient for the detection of early onset movement and cannot quantitatively measure pelvic rotation. The pressure biofeedback unit (PBU) is already commonly used in clinical settings to detect and retrain pelvic movement patterns during lower limb movements, ${ }^{12-14}$ and should therefore be investigated as an alternative tool to measure pelvic movement during hip flexion.

Tightness of the posterior hip muscles or joint capsule can cause excessive substitutive pelvic motion in the sagittal plane during hip flexion. ${ }^{15}$ Specifically, because the proximal attachment of hamstrings occurs at the ischial tuberosity, hamstring tension influences pelvic movement during hip flexion. ${ }^{7}$ Previous studies have reported that the contribution of posterior pelvic tilting to hip flexion is greater in individuals with shorter hamstrings, and in knee extension rather than flexion. ${ }^{7,8}$ However, no study has yet investigated changes in pelvifemoral rhythm during hip flexion following hamstring stretching. If pelvic movement can be measured indirectly by a PBU during hip flexion, then the relationship between changes in biofeedback pressure recorded by the PBU and the pelvic angle accompanying hip flexion after hamstring stretching could be examined.

Numerous studies have examined pelvic rotation following hip flexion in various postures, including supine, ${ }^{4-7,9}$ standing upright, ${ }^{10}$ and hanging by the hands from a bar. ${ }^{8}$ Hip flexion maneuvers have been compared between passive and active motion, between unilateral and bilateral motion, and between knee extension and flexion. ${ }^{4-10}$ In the current study, we examined hip flexion during passive straight leg raising (PSLR) because PSLR is commonly used as a clinical test of hamstring muscle length. Therefore, the objectives of this study were to 1) determine the relationship between pelvic motion and biofeedback pressure measured by a PBU during PSLR and 2) identify changes in biofeedback pressure during PSLR immediately following stretching in subjects with short hamstrings. We hypothesized 1) a positive relationship between pelvic rotation and biofeedback pressure and 2) decreased biofeedback pressure during PSLR after hamstring stretching in subjects with short hamstring.

\section{METHODS}

\section{Participants}

Twenty young adults (14 men and 6 women; mean age,
$23.95 \pm 1.96$ years; mean body height, $168.25 \pm 7.69 \mathrm{~cm}$; mean body mass, $66.6 \pm 10.46 \mathrm{~kg}$ ) with short hamstring muscles participated in this study. The sole inclusion criterion was $>20^{\circ}$ bilateral knee extension, as measured by the active knee extension (AKE) test. ${ }^{16}$ The following exclusion criteria were applied: (1) lower back pain, (2) history of lower limb pain or previous hamstring injury, and (3) participation in sports or any specific exercise program requiring lengthening of the hamstring within 3 months prior to the study. The sample size was calculated using G*Power software. We used a two-tailed t-test for dependent samples $(\alpha=0.05$, effect size $=0.7$ ) to determine that a sample size of 20 was required to achieve $90 \%$ power. This study was approved by the Institutional Review Board (IRB) of Joongbu University. All participants were verbally informed of the experimental procedure, objectives, and risks of the study, and provided written informed consent prior to participation.

\section{Instrumentation}

A digital goniometer (Dualer IQ; J Tech Medical Industries, Heber City, UT, USA) was used to perform the AKE test, and during PSLR in $10^{\circ}$ increments. We used a CMS20 ultrasound-based motion analysis system (Winspine for triple lumbar; Zebris Medizintechnik GmbH, Isny, Germany) to measure the pelvic angle in real time during PSLR. This three-dimensional (3D) motion equipment consists of two triple ultrasound transmitters with belt attachments and a fixed system of three microphones. The pelvic angle was calculated by using the microphones to determine the spatial coordinates of the ultrasound transmitters, at a sampling rate of $50 \mathrm{~Hz}$, using the Winspine 2.22 software. This system has been validated in several spinal motion studies. ${ }^{17-19}$ A PBU (Stabilizer Pressure Biofeedback Unit, Chattanooga Group Inc., Hixson, TN, USA) was used to determine changes in pressure in the lumbopelvic region during PSLR before and after hamstring stretching.

\section{Hamstring length measurement}

All measurements were conducted with participants in a supine position on a wooden table. Only the right leg was tested. Before and after hamstring stretching, the AKE test was performed to determine changes in hamstring length. ${ }^{19}$ Subjects were placed in the supine position, with the hip and knee flexed at $90^{\circ}$ and the anterior thigh touching a cross-bar to maintain hip flexion. A digital inclinometer was attached below the fibular head using an elastic strap. The starting position was calibrated to $0^{\circ}$ at $90^{\circ}$ knee flexion in the horizontal position. Subjects were then asked to actively extend the leg until the point of limitation, keeping the feet relaxed without ankle dorsiflexion and the thigh against the 
bar. The AKE angle (degree of knee extension relative to initial knee flexion) was recorded again in the final position. The AKE angle was defined as the degree of knee flexion from complete knee extension. AKE angles were recorded in triplicate before and after hamstring stretching.

\section{Measurements of pelvic angle and biofeedback pressure}

The experimental setting showed in Figure 1. To measure pelvic angle and biofeedback pressure during PSLR before and after hamstring stretching, we mounted the motion analyzer microphones at a distance of $80 \mathrm{~cm}$ from the measured leg. A digital goniometer was attached with a strap halfway along the femur shaft to conduct measurements during PSLR at $10^{\circ}$ increments until hip joint flexion reached $70^{\circ}$ (Figure 1). ${ }^{20}$ To measure the pelvic angle, one belt holding a triple ultrasound transmitter was attached firmly at the thoracolumbar junction to isolate pelvic movement, and another was attached at the level of the anterior superior iliac and posterior superior iliac spines. ${ }^{16}$ A PBU airbag was placed under the lumbar spine (L1-S1), centered at the L3 spinous process above L4, and was palpated between the tops of the left and right iliac crests. The airbag was inflated to a base pressure of $40 \mathrm{mmHg}$ and the pelvic angle was calibrated to $0^{\circ}$ in the supine position. The experimenter then raised the straight leg in $10^{\circ}$ increments during PSLR, from $0^{\circ}$ to $70^{\circ}$, at a movement velocity of approximately $5^{\circ} \mathrm{s}^{-1}$. Each position was held for $5 \mathrm{~s}$, while biofeedback pressure and pelvic angle were recorded. Subjects feeling pain during PSLR were not asked to raise the leg further. ${ }^{20}$ To determine the test-retest reliability of PBU measurements, we followed the same procedure with 10 subjects, 2 weeks prior to the study.

\section{Hamstring stretching}

Hamstring stretching was performed twice; AKE stretching

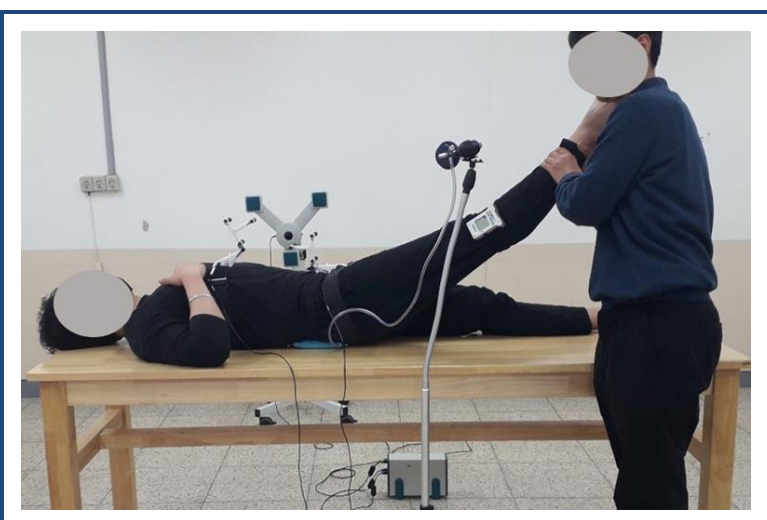

Figure 1. Measurement of pelvic angle and biofeedback pressure during PSLR (passive straight leg raising). was always performed before PSLR stretching. Stretching lasted approximately 3 minutes. This static stretching was repeated three times with 10 -s rest periods between each stretch; stretching positions were held for $15 \mathrm{~s}$. Hamstring stretching was performed and monitored by the primary experimenter. The subject was asked to relax the lower extremity during stretching, and verbal feedback was provided throughout the stretching protocol to ensure that the lumbar spine and pelvis were maintained in a neutral position.

To perform AKE stretching, each subject was asked to actively and gradually extend the lower leg in the AKE test position until as much stretching force as possible was applied without pain. Once this position was achieved, the subject held it for $15 \mathrm{~s}$. PSLR stretching was performed with the experimenter standing beside the dominant leg, slowly raising the leg perpendicular to the planar surface with one hand placed under the ankle joint and the other hand placed above the knee joint. The subject was asked to inform the primary experimenter when strong but tolerable discomfort was felt during the stretch. The lower extremity was then lowered by a few degrees from this symptomatic position.

\section{Statistical analyses}

The test-retest reliability of PBU measurements was assessed using interclass correlation coefficients $\left(\mathrm{ICC}_{3,1}\right)$ (95\% confidence interval [CI]) and standard error of measurement (SEM). Degree of reliability bases on ICCs was defined as following: $<0.70=$ poor, $0.70-0.79=$ moderate, $0.80-.89=$ good, and $0.90-0.99=$ excellent. Pearson product moment correlations were used to describe the relationships among PSLR angle, biofeedback pressure, and pelvic angle. Simple linear regression analysis was performed to predict biofeedback pressure according to the pelvic angle. Paired ttests were used to determine significant differences in AKE angle, biofeedback pressure, and pelvic angle, using the PSLR angle before and after hamstring stretching. Statistical significance was determined at a level of $p<0.05$. Data were analyzed using SPSS software (ver. 10.0; SPSS Inc., Chicago, IL, USA).

\section{RESULTS}

Test-retest reliability analysis showed excellent reliability for PBU measurements $\left(\mathrm{ICC}_{3,1}=0.94-0.99\right.$; SEM=0.47-0.84 $\mathrm{mmHg}$ ). There was a significant relationship between pelvic angle and PSLR angle (Pearson's $r=0.75, p<0.05$ ), between biofeedback pressure and PSLR angle (Pearson's $r=0.80$, $p<0.05$ ), and between pelvic angle and biofeedback pressure 
(Pearson's $r=0.76, p<0.05$ ) (Figure 2 and 3). The linear regression equation for pelvic angle as an independent factor $\left(\mathrm{R}^{2}=0.57, p<0.05\right)$ was as follows:

\section{Biofeed lback pressure $=45.03+(3.35 \times$ pelvic angle $)$}

The AKE angle was significantly less post-stretch (mean \pm standard deviation $\left.[\mathrm{SD}]=27.77^{\circ} \pm 6.16^{\circ}\right)$ than pre-stretch $\left(\right.$ mean $\left.\pm \mathrm{SD}=36.85^{\circ} \pm 6.68^{\circ}\right) \quad(p<0.01)$. Biofeedback pressure decreased significantly within a PSLR range of $10-60^{\circ}$, and pelvic angle decreased significantly at PSLR angles of $50^{\circ}$ and $60^{\circ}$ after hamstring stretching $(p<0.05)$ (Table 1$)$.

\section{DISCUSSION}

The present study obtained clinically acceptable reliability values for PBU pressure measurements during PSLR $\left(\mathrm{ICC}_{3,1}=0.94-0.99\right)$. There was a significant linear relationship between biofeedback pressure and pelvic angle. These findings indicate that biofeedback pressure can be measured reliably as result of pelvic movement during PSLR. Azevedo et al. ${ }^{12}$ reported intra-rater reliability values for biofeedback pressure during AKE of 0.75, 0.77, and 0.79 at $30^{\circ}, 60^{\circ}$, and $90^{\circ}$ knee flexion, respectively. The higher reliability values found in the current study can be explained by the type of lower limb movement examined; the previous study measured biofeedback pressure during active movement to assess lumbopelvic stability, whereas the current study measured biofeedback pressure during passive movement to test hamstring muscle length. Biofeedback pressure measurements may be more reliable during PSLR than during AKE testing because the lumbopelvic movements change with subject's effort during AKE testing.

The significant linear relationship obtained between pelvic and PSLR angles in this study was consistent with the results of previous studies describing pelvifemoral rhythm. Previous studies have reported that the mean contribution of the pelvic angle to total hip flexion ranged from $8.0 \%$ to $37.5 \%$. PSLR was performed as a hip flexion task in two such studies, which reported that the pelvic angle contributed $22.9^{\circ}(26.8 \%)$ to a total PSLR angle of $85.5^{\circ} 4$ and $32.1^{\circ}$ (36.9\%) to a total PSLR angle of $87.3^{\circ} .5$ Our pelvifemoral rhythm results showed that pelvic motion contributed $8.39^{\circ}$

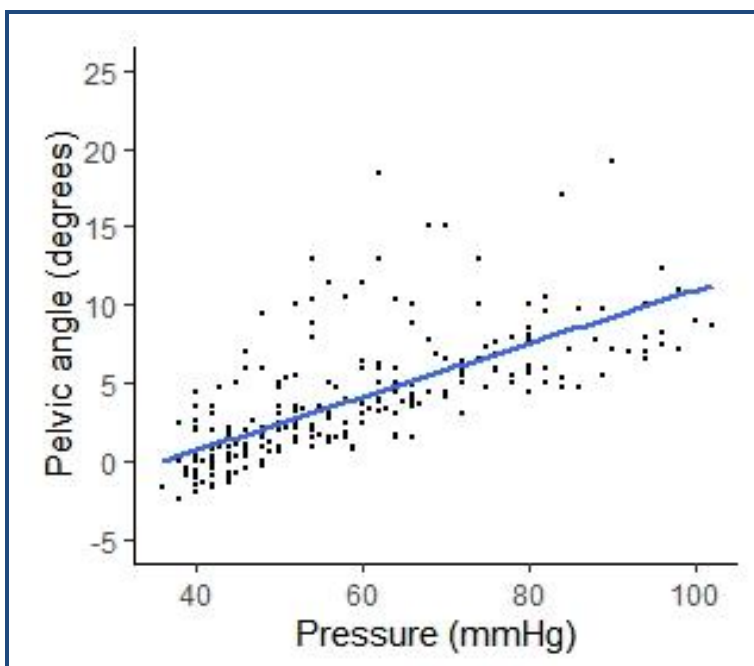

Figure 3. Linear relationship between biofeedback pressure and pelvic angle during PSLR (passive straight leg raising).

A. B.
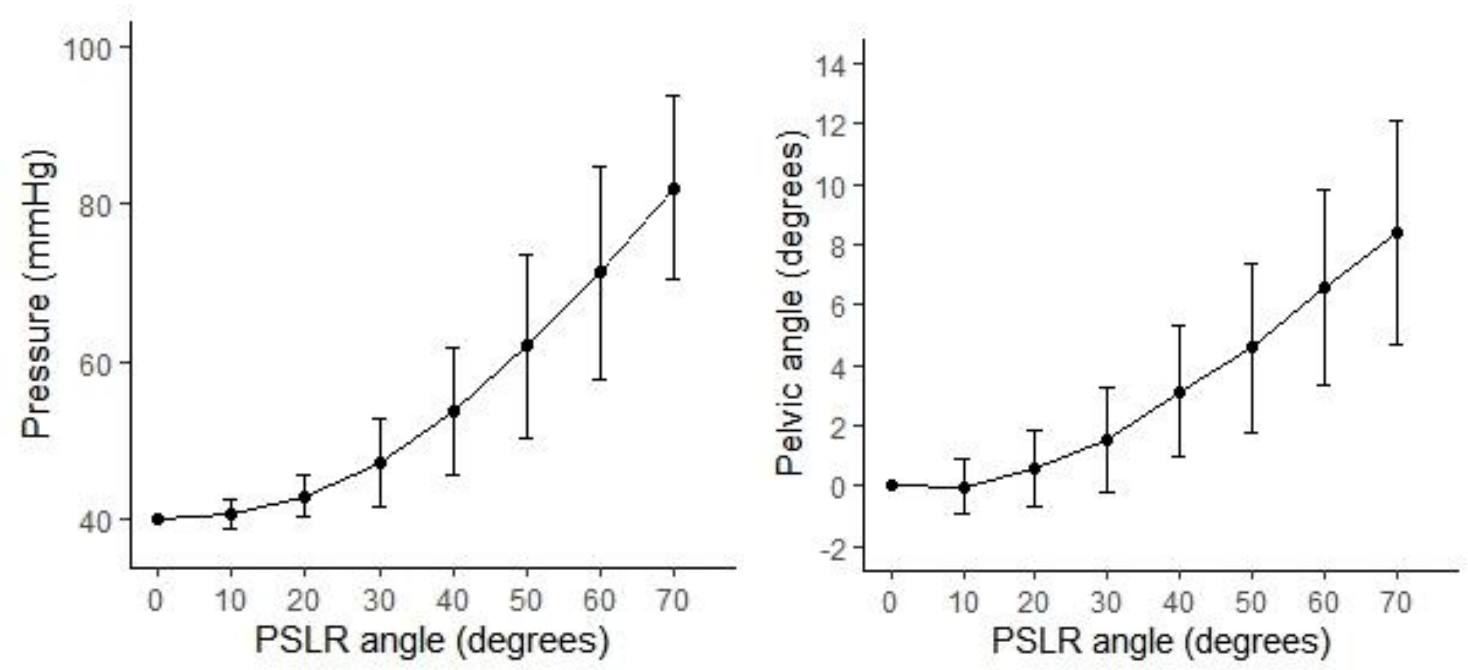

Figure 2. Graph of pressure of PBU (A) and pelvic rotation (B) accompanying PSLR (passive straight leg raising). 
Table 1. Data in biofeedback pressure and pelvic angle according to PSLR angle before and after hamstring stretching

\begin{tabular}{|c|c|c|c|c|c|c|c|c|}
\hline \multirow{2}{*}{$\begin{array}{l}\text { PSLR } \\
\text { Angle }\end{array}$} & \multicolumn{4}{|c|}{ Pressure of PBU } & \multicolumn{4}{|c|}{ Pelvic angle } \\
\hline & Pre-stretch & Post-stretch & $p$ & Effect size & Pre-stretch & Post-stretch & $p$ & Effect size \\
\hline $10^{\circ}$ & $41.05 \pm 1.88$ & $40.40 \pm 2.01$ & 0.012 & 0.63 & $0.01 \pm 0.86$ & $-0.02 \pm 1.01$ & 0.935 & 0.02 \\
\hline $20^{\circ}$ & $43.40 \pm 2.80$ & $42.40 \pm 2.54$ & 0.007 & 0.67 & $0.63 \pm 1.32$ & $0.49 \pm 1.22$ & 0.527 & 0.14 \\
\hline $30^{\circ}$ & $48.75 \pm 6.26$ & $45.70 \pm 4.74$ & 0.018 & 0.58 & $1.68 \pm 1.81$ & $1.38 \pm 1.66$ & 0.461 & 0.17 \\
\hline $40^{\circ}$ & $55.25 \pm 8.35$ & $52.20 \pm 7.59$ & 0.010 & 0.64 & $3.44 \pm 2.35$ & $2.85 \pm 2.04$ & 0.072 & 0.43 \\
\hline $50^{\circ}$ & $64.30 \pm 12.22$ & $12.22 \pm 10.76$ & 0.005 & 0.71 & $4.99 \pm 3.05$ & $4.16 \pm 2.48$ & 0.037 & 0.50 \\
\hline $60^{\circ}$ & $73.30 \pm 13.66$ & $69.35 \pm 13.26$ & 0.041 & 0.49 & $7.21 \pm 3.50$ & $5.98 \pm 2.93$ & 0.005 & 0.71 \\
\hline $70^{\circ}$ & $83.44 \pm 13.29$ & $80.75 \pm 10.21$ & 0.147 & 0.38 & $9.39 \pm 4.29$ & $7.39 \pm 2.78$ & 0.050 & 0.53 \\
\hline
\end{tabular}

Data are presented as means \pm standard deviation. *Asterisks indicate significant differences determined by paired $t$-tests.

PSLR - passive straight leg raising; PBU - pressure biofeedback unit.

$(11.99 \%)$ to a total PSLR angle of $70^{\circ}$, which was calculated using mean values obtained before and after hamstring stretching. We infer that the contribution of pelvic movement to total PSLR excursion in the current study was less than that reported in previous studies for two reasons. First, due to differences in total PSLR excursion, pelvic angles reported in previous studies were greater than that determined in this study. Second, previous studies did not isolate pelvic movement from movement of the lower thoracic region, whereas we measured pelvic movement selectively, by attaching triple markers to the thoracolumbar junction.

The hypothesis of this study was that biofeedback pressure would increase with posterior tilting of the pelvis and flattening of the lumbar spine in the sagittal plane during PSLR. We detected a positive linear relationship between pelvic angle and biofeedback pressure in subjects with short hamstring. As similar to this study, a previous study a positive linear relationship between pelvic angle and biofeedback pressure in subjects with normal hamstring. ${ }^{20}$ PSLR is a clinical test commonly used to measure hamstring muscle length. Our findings indicate that biofeedback pressure measured by a PBU can be employed to measure pelvic movement during hamstring muscle length assessments, for example when the cross-sectional area of the muscle is used as an indicator of muscle strength. According to our regression equation, an increase in pelvic movement by $1^{\circ}$ would lead to an increase in biofeedback pressure of $3.35 \mathrm{mmHg}$. For example, a $5^{\circ}$ increase in pelvic angle produces a biofeedback pressure estimate of $62 \mathrm{mmHg}$.

Previous studies have reported that the contribution of pelvic tilting to hip flexion begins very early and is continuous throughout the range of hip flexion, similar to the contribution of scapular upward rotation to shoulder elevation. ${ }^{5-8,10,11}$ In the current study, both biofeedback pressure and pelvic angle began to increase after $10^{\circ}$ of PSLR in most subjects. As previously reported, ${ }^{21-23}$ the results of the current study suggest that measuring the range of hip flexion as the thigh angle relative to the horizontal plane at the point of perceived onset of the lumbopelvic movement is inappropriate. Thus, clinicians need to be aware of the concurrent pelvifemoral rhythm when measuring the range of hip flexion.

Hamstring flexibility has been proposed as a predisposing factor for non-specific low back pain. ${ }^{24}$ Tight hamstrings can restrict hip motion, thereby increasing lumbopelvic motion and causing back pain due to its influence on lumbopelvic rhythm during forward bending. ${ }^{25}$ Although several studies have reported the immediate effects of hamstring stretching on range of motion, and a reduction in tension and stiffness in the muscle-tendon unit, ${ }^{26-28}$ no studies have determined the effect of hamstring stretching on pelvifemoral rhythm during PSLR. Our results show that pelvic motion contributed $9.39^{\circ}(13.41 \%)$ and $7.39^{\circ}$ $(10.56 \%)$ to a total PSLR angle of $70^{\circ}$ before and after hamstring stretching, respectively. These findings demonstrate that hamstring stretching immediately before and after PSLR changed the pelvifemoral rhythm by decreasing pelvic motion during PSLR.

This change in pelvifemoral rhythm was associated with a decrease in biofeedback pressure during PSLR after hamstring stretching. Improved hamstring flexibility decreased the posterior tilting of the pelvis and lumbar spine flexion. The PBU airbag under the lumbopelvic region received less pressure during PSLR after stretching than before. Therefore, change in hamstring length can be indirectly detected based on pelvic angle and biofeedback pressure, as measured by a PBU during PSLR. Biofeedback pressure decreased significantly at a PSLR angle range of 
$10-60^{\circ}$; however, pelvic angle decreased significantly only at PSLR angles of $50^{\circ}$ and $60^{\circ}$ after hamstring stretching. This result indicates that biofeedback pressure is more sensitive than pelvic angle to changes in PSLR angle after hamstring stretching.

There were some limitations to this study. First, we did not exclude other factors affecting the pelvifemoral rhythm during PSLR. In addition to hamstring flexibility, pelvic motion and biofeedback pressure can be influenced by stiffness in single-joint muscles (e.g., gluteus maximus) and the posterior capsule and ligament around the hip joint. Second, biofeedback pressure values were recorded from the PBU without normalization. To compare PBU measurements between individuals, a normalization procedure is required. Future studies must develop normalization protocols for PBU measurements obtained during PSLR. Finally, the results of our study cannot be generalized because only young subjects with short hamstrings participated without control group. Therefore, it is needed to compare the difference of biofeedback pressure and pelvic angle during should be investigated between subjects with and without hamstring stretching in future study.

\section{CONCLUSION}

We detected a positive linear relationship between biofeedback pressure measured by a PBU and pelvic angle during PSLR in subjects with short hamstrings. Immediate hamstring stretching tended to decrease pelvic angle and biofeedback pressure during PSLR. Therefore, we recommend the use of a PBU to indirectly measure the degree of pelvic motion when testing hamstring length after stretching.

\section{Key Points}

Question Are there relationship between pelvic motion and biofeedback pressure measured by a PBU during PSLR, and changes in biofeedback pressure after hamstring stretching?

Findings There was a significant positive relationship between pelvic motion and biofeedback pressure. Biofeedback pressure and pelvic angle decreased significantly in some PSLR angle after hamstring stretching.

Meaning Biofeedback pressure measured with a PBU can be used to assess pelvic motion during muscle length tests.

\section{Article information}

Conflict of Interest Disclosures: None.

Funding/Support: This paper was supported by Joongbu University Research \& Development Fund, in 2021.
Acknowledgment: None.

Ethic Approval: This study was approved by the Institutional Review Board (IRB) of Joongbu University.

\section{REFERENCES}

1. Norkin CC, White DJ. Measurement of joint motion: a guide to goniometery. 3rd Spiral ed. F.A. Davis Company; 2003.

2. Zook L, Brittis D, Gleim G. A new pelvic tilt detection device: roentgenographic validation and application to assessment of hip motion in professional ice hockey players. J Orthop Sports Phys Ther. 1996;24(5):303-308.

3. Gajdosik RL, Rieck MA, Sullivan DK, Wightman SE. Comparison of four clinical tests for assessing hamstring muscle length. J Orthop Sports Phys Ther. 1993;18(5): 614-618.

4. Bohannon RW. Cinematographic analysis of the passive straight-leg-raising test for hamstring muscle length. Phys Ther. 1982;62:1269-174.

5. Bohannon R, Gajdosik R, LeVeau BF. Contribution of pelvic and lower limb motion to increases in the angle of passive straight leg raising. Phys Ther. 1985;65:474-476.

6. Bohannon RW, Gajdosik RL, LeVeau BF. Relationship of pelvic and thigh motions during unilateral and bilateral hip flexion. Phys Ther. 1985;65:1501-1504.

7. Congdon R, Bohannon R, Tiberio D. Intrinsic and imposed hamstring length influence posterior pelvic rotation during hip flexion. Clin Biomech (Bristol, Avon). 2005; 20:947-941.

8. Dewberry MJ, Bohannon RW, Tiberio D, Murray R, Zannotti CM. Pelvic and femoral contributions to bilateral hip flexion by subjects suspended from a bar. Clin Biomech (Bristol, Avon). 2003;18:494-499.

9. Elia DS, Bohannon RW, Cameron D, Albro RC. Dynamic pelvic stabilization during hip flexion: a comparison study. J Orthop Sports Phys Ther. 1996;24:30-36.

10. Murray R, Bohannon R, Tiberio D, Dewberry M, Zannotti C. Pelvifemoral rhythm during unilateral hip flexion in standing. Clin Biomech (Bristol, Avon). 2002; 17:147-1451.

11. Van Houcke J, Pattyn C, Vanden Bossche L, Redant C, Maes JW, Audenaert EA. The pelvifemoral rhythm in cam-type femoroacetabular impingement. Clin Biomech (Bristol, Avon). 2014;29:63-67.

12. Azevedo DC, Lauria AC, Pereira AR, et al. Intraexaminer and interexaminer reliability of pressure biofeedback unit for assessing lumbopelvic stability during 6 lower limb movement tests. J Manipulative Physiol Ther. 2013;36(1):33-43. 
13. Cairns MC, Harrison K, Wright C. Pressure biofeedback: a useful tool in the quantification of abdominal muscular dysfunction?. Physiotherapy. 2000;86:127-138.

14. Richardson C, Jull G, Hodges P, Hides JA. Therapeutic exercise for spinal segmental stabilization in low back pain. in: scientific basis and clinical approach. Churchill Livingstone: Edinburgh; 1999.

15. Kim SH, Kwon OY, Yi CH, Cynn HS, Ha SM, Park KN. Lumbopelvic motion during seated hip flexion in subjects with low-back pain accompanying limited hip flexion. Eur Spine J. 2014; 23(1):142-148.

16. Sullivan MK, Dejulia JJ, Worrell TW. Effect of pelvic position and stretching method on hamstring muscle flexibility. Med Sci Sports Exerc. 1992;24(12):13831389.

17. Moutzouri M, Billis E, Strimpakos N, Kottika P, Oldham JA. The effects of the Mulligan Sustained Natural Apophyseal Glide (SNAG) mobilisation in the lumbar flexion range of asymptomatic subjects as measured by the Zebris CMS20 3-D motion analysis system. BMC Musculoskelet Disord. 2008;1(9):131.

18. Dvir Z, Prushansky T. Reproducibility and instrument validity of a new ultrasonography-based system for measuring cervical spine kinematics. Clin Biomech (Bristol, Avon). 2000;15(9):658-664.

19. Malmström EM, Karlberg M, Melander A, Magnusson M. Zebris versus Myrin: a comparative study between a three-dimensional ultrasound movement analysis and an inclinometer/compass method: intradevice reliability, concurrent validity, intertester comparison, intratester reliability, and intraindividual variability. Spine (Phila Pa 1976). 2003;28(21):E433-440.

20. Jung DY. The correlation of the pressure of biofeedback unit and lumbopelvic motion during straight leg raising in healthy subjects. Phys Ther Korea. 2018;25(3):12-18.

21. Kuo YL, Tully EA, Galea MP. Lumbofemoral rhythm during active hip flexion in standing in healthy older adults. Man Ther. 2010;15:88-92.

22. Bohannon RW, Bass A. Research describing pelvifemoral rhythm: a systematic review. J Phys Ther Sci. 2017; 29(11):2039-2043.

23. Tully EA, Wagh P, Galea MP. Lumbofemoral rhythm during hip flexion in young adults and children. Spine. 2002;27:E432-E440.

24. Esola MA, McClure PW, Fitzgerald GK, Siegler S. Analysis of lumbar spine and hip motion during forward bending in subjects with and without a history of low back pain. Spine (Phila Pa 1976). 1996;21(1):71-78.

25. Jones MA, Stratton G, Reilly T, Unnithan VB. Biological risk indicators for recurrent non-specific low back pain in adolescents. Br J Sports Med. 2005;39(3):137140.

26. O'Hora J, Cartwright A, Wade CD, Hough AD, Shum GL. Efficacy of static stretching and proprioceptive neuromuscular facilitation stretch on hamstrings length after a single session. $J$ Strength Cond Res. 2011;25(6): 1586-1591.

27. Puentedura EJ, Huijbregts PA, Celeste S, Edwards D, In A, Landers MR, Fernandez-de-Las-Penas C. Immediate effects of quantified hamstring stretching: hold-relax proprioceptive neuromuscular facilitation versus static stretching. Phys Ther Sport. 2011;12(3):122-126.

28. Youdas JW, Haeflinger KM, Kreun MK, Holloway AM, Kramer CM, Hollman JH. The efficacy of two modified proprioceptive neuromuscular facilitation stretching techniques in subjects with reduced hamstring muscle length. Physiother Theory Pract. 2010;26(4):240-250. 